\title{
TITLE:
}

\section{Lattice polyhedra and submodular flows}

\author{
$\operatorname{AUTHOR}(\mathrm{S})$ :
}

Fujishige, Satoru; Peis, Britta

CITATION:

Fujishige, Satoru ...[et al]. Lattice polyhedra and submodular flows. Japan Journal of Industrial and Applied Mathematics 2012, 29(3): 441451

ISSUE DATE:

2012-10

URL:

http://hdl.handle.net/2433/167739

\section{RIGHT:}

The final publication is available at www.springerlink.com; この論文は 出版社版でありません。引用の際には出版社版をご確認ご利用くださ $\omega_{\circ}$; This is not the published version. Please cite only the published version. 


\title{
Lattice Polyhedra and Submodular Flows
}

\author{
Satoru Fujishige · Britta Peis
}

Received: date / Accepted: date

\begin{abstract}
Lattice polyhedra, as introduced by Gröflin and Hoffman, form a common framework for various discrete optimization problems. They are specified by a lattice structure on the underlying matrix satisfying certain sub- and supermodularity constraints. Lattice polyhedra provide one of the most general frameworks of total dual integral systems. So far no combinatorial algorithm has been found for the corresponding linear optimization problem. We show that the important class of lattice polyhedra in which the underlying lattice is of modular characteristic can be reduced to the Edmonds-Giles polyhedra. Thus, submodular flow algorithms can be applied to this class of lattice polyhedra. In contrast to a previous result of Schrijver, we do not explicitly require that the lattice is distributive. Moreover, our reduction is very simple in that it only uses an arbitrary maximal chain in the lattice.
\end{abstract}

Keywords Lattice polyhedra $\cdot$ Edmonds-Giles polyhedra $\cdot$ distributive lattices

\section{Introduction}

Discrete optimization problems often allow, or even ask for, a formulation as an integer linear program, i.e., as a problem to find optimal integral solutions of a linear program of type

$$
\max \left\{w^{T} x \mid A x \leq f\right\},
$$

where $A \in\{-1,0,1\}^{m \times n}, w \in \mathbb{R}^{n}$ and $f \in \mathbb{R}^{m}$. By duality theory, the value of the linear problem is equal to the value of its dual linear program $\min \left\{y^{T} f \mid y^{T} A=w^{T}, y \geq\right.$ $0\}$.

Satoru Fujishige

Research Institute for Mathematical Sciences, Kyoto University, Japan

E-mail: fujishig@kurims.kyoto-u.ac.jp

Britta Peis

TU Berlin, Straße des 17. Juni 136, 10623 Berlin, Germany.

E-mail: peis@math.tu-berlin.de 
Being interested in integral solutions, a fundamental problem in polyhedral combinatorics is to decide which linear programs have guaranteed integer optimal solutions. The probably most important concept here is that of total dual integral inequality systems [22], where system $A x \leq f$ is called total dual integral (TDI) if the minimum in $\min \left\{y^{T} f \mid y^{T} A=w^{T}, y \geq 0\right\}$ can be achieved by an integral vector $y$ for each integral $w$ for which the optima exist. Various classes of linear programs with specially structured right-hand sides (often submodular) can be proven to have integral optimal solutions. However, the development of polynomial algorithms to solve TDI problems has lagged behind the theoretical results, although there have been some recent successes (see, e.g., [16], [17], [18].)

When asking for combinatorial algorithms for as general TDI-systems as possible, one promising model to look at is that of lattice polyhedra which were introduced by Hoffman and Schwartz [12] for $\{0,1\}$-matrices, and generalized by Gröflin and Hoffman [11] to $\{-1,0,1\}$-matrices.

\subsection{Lattice polyhedra}

The name "lattice polyhedron" comes from a certain, very general, lattice structure on the underlying matrix on which the right-hand side is submodular. We first need some notation: A poset $(L, \preceq)$ is a lattice if for any two elements $i, j \in L$ there exist a "meet" $i \wedge j=\sup \{k \in L \mid k \preceq i, j\}$ and a "join" $i \vee j=\inf \{k \in L \mid k \succeq i, j\}$. We denote by $m$ and $M$ the (unique) minimal and maximal element of lattice $L$, respectively. A function $f: L \rightarrow \mathbb{R}$ is submodular if it satisfies for all $i, j \in L$ the inequality $f(i)+$ $f(j) \geq f(i \wedge j)+f(i \vee j)$.

Definition 1 [Lattice polyhedron [11]] Let $(L, \preceq, \wedge, \vee)$ be a lattice on which $f \in \mathbb{R}^{|L|}$ is submodular. Given a matrix $A \in\{-1,0,1\}^{|L| \times|E|}$ with entries $\chi(i, e)$ for $i \in L$ and $e \in E$, lower and upper bounds $c, d \in \mathbb{R}^{|E|}$ the polyhedron

$$
\mathbb{P}(A, f)=\left\{x \in \mathbb{R}^{|E|} \mid A x \leq f, c \leq x \leq d\right\}
$$

is called a lattice polyhedron if for all $i, j, k \in L$ and all $e \in E$ the following three hold:

(C1) if $i \prec j \prec k$ and $\chi(i, e)=\chi(k, e)=t \neq 0$, then $\chi(j, e)=t$,

(C2) if $i \prec j$, then $\chi(i, e) \cdot \chi(j, e) \geq 0$, and

(C3) $\chi(i, e)+\chi(j, e) \leq \chi(i \vee j, e)+\chi(i \wedge j, e)$.

Analogously, if, in the above definition, function $f$ is supermodular (i.e., if $-f$ is submodular) and $(\mathrm{C} 3)$ is replaced by $\left(\mathrm{C}^{\prime}\right) \chi(i, e)+\chi(j, e) \geq \chi(i \vee j, e)+\chi(i \wedge j, e)$, the polyhedron $\mathbb{P}^{\prime}(A, f)=\left\{x \in \mathbb{R}^{|E|} \mid A x \geq f, c \leq x \leq d\right\}$ is also called a lattice polyhedron. The pair of the matrix $A$ and the lattice $L$ is called consecutive if properties $(\mathrm{C} 1)$ and $(\mathrm{C} 2)$ are satisfied. If $(A, L)$ satisfies $(\mathrm{C} 3)$ (or $\left(\mathrm{C}^{\prime}\right)$, or both), we say that $(A, L)$ has supermodular (or submodular, or modular) characteristic. For technical reasons, we assume throughout that for each $e \in E$ there exists at least one $i \in L$ such that $\chi(i, e) \neq 0$.

Lattice polyhedra form a common framework for various combinatorial structures. For example, lattice polyhedra with $\{0,1\}$-matrices include polyhedra types 
such as polymatroids, the intersection of polymatroids, and submodular systems. The lattice polyhedra with $\{-1,0,1\}$-matrix include Edmonds-Giles polyhedra [3], so that the optimization problem for lattice polyhedra also covers the well-studied submodular flow problem. (See Section 2 below for the definition of Edmonds-Giles polyhedra and the submodular flow problem). Several min-max results for combinatorial structures can be derived from the following theorem:

Theorem 1 ([11]) If either $(A, L)$ is of modular characteristic, or $c \geq 0$, then the system of linear inequalities for the lattice polyhedron is totally dual integral. If $f, c$ and $d$ are integral, then all vertices of lattice polyhedra are integral.

However, Gröflin and Hoffman's integrality result for lattice polyhedra is only a structural existence theorem without algorithmic foundation. In the last decades, combinatorial algorithms have been developed only for various special instances of lattice polyhedra. For example, there exist several greedy-type algorithms for special classes of $\{0,1\}$-lattice polyhedra (e.g., [7], [6], [2], [4], [5], [13], [15]), as well as a primal-dual algorithm for $\{0,1\}$-lattice polyhedra with supermodular right-hand side [18].

Lattice polyhedra on $\{-1,0,1\}$-matrices seem to be a lot harder to investigate under algorithmic aspects. For $\{-1,0,1\}$-lattice polyhedra, algorithms have mainly been developed for the special case of Edmonds-Giles polyhedra. (See e.g. [20], [14], [19] for the currently fastest submodular flow algorithms, or the survey paper [10].) The reason why polyhedra on $\{-1,0,1\}$-matrices and their underlying structure are not so tractable might be that it is much more natural to play around with subset families $L \subseteq 2^{E}$ than with families of disjoint ordered tuples $L \subseteq 3^{E}=\{(X, Y) \mid X, Y \subseteq$ $E, X \cap Y=\emptyset\}$ in which the elements may occur "positive" or "negative". Thus, in order to design a combinatorial algorithm for $\{-1,0,1\}$-lattice polyhedra in general, we would need to better understand the underlying combinatorial structure, and maybe even give some graph-theoretic interpretation of the problem. Moreover, we would need to find an appropriate oracle to access the lattice.

Our contribution. This paper can be seen as a further step toward this goal. We will show that $\{-1,0,1\}$-lattice polyhedra in which $(A, L)$ is of modular characteristic can be reduced to Edmonds-Giles polyhedra via a very simple reduction. For our reduction, we only need an oracle that gives us an arbitrary maximal chain in $L$. In contrast to a related result of Schrijver [21], we do not require explicitly that $L$ is a distributive lattice, and we do not need an oracle to return minimal and maximal elements in certain sublattices. The differences between Schrijver's and our reduction will be described below.

\section{Preliminaries and Definitions}

Let $\mathbb{P}(A, f)=\left\{x \in \mathbb{R}^{|E|} \mid A x \leq f, c \leq x \leq d\right\}$ be a lattice polyhedron on lattice $L=$ $(L, \preceq, \wedge, \vee)$. Thus, by Definition 1 , the pair $(A, L)$ is consecutive and of supermodular characteristic, and $f$ is submodular. 
Note that, since $A$ is a $\{-1,0,1\}$-matrix, we might as well identify each $i \in L$ with some element $\left(X_{i}, Y_{i}\right)$ in $3^{E}$, where $3^{E}$ denotes the family of all ordered pairs $(X, Y)$ of disjoint subsets of a finite set $E$, i.e., $A$ can be interpreted as the incidence matrix of some (multi-)set of elements in $3^{E}$ via

$$
\chi(i, e)=\left\{\begin{aligned}
1 & \text { if } e \in X_{i} \\
-1 & \text { if } e \in Y_{i} \\
0 & \text { otherwise }
\end{aligned}\right.
$$

With this interpretation, $\mathbb{P}(A, f)$ can equivalently be written as $\mathbb{P}(L, f)=\left\{x \in \mathbb{R}^{E}\right.$ $\left.\forall i \in L: x\left(X_{i}\right)-x\left(Y_{i}\right) \leq f_{i}, c \leq x \leq d\right\}$. To shorten notation, we call lattice $L$ consecutive, supermodular, or modular if the pair $(A, L)$ is consecutive, supermodular, or modular, respectively. For each $i \in L$ we denote by $\chi(i)$ the $\{-1,0,1\}$-vector $(\chi(i, e) \mid e \in E)$. For technical reasons, we assume that $\chi(m)=\chi(M)=\emptyset$ and $f(m)=$ $f(M)=0$ for the unique minimal and maximal element $m$ and $M$ in $L$, respectively.

As usual, we assume that the size of $E$ and the height of the lattice (i.e., the size of a maximal chain in $L$ ) are rather small, whereas the size of the lattice $L$ might be huge, say exponential in $|E|$. These assumptions are justified by all the known examples and applications of lattice polyhedra (see, e.g., [21]). The function values of $f$ are assumed to be given implicitly via some oracle.

Chains, ideals, lower neighbours, join-irreducible elements. Given a poset $(P, \preceq)$, a subset $C \subseteq P$ is a chain, if for any two elements $i, j \in C$ either $i \preceq j$ or $j \preceq i$. A subset $I \subseteq P$ is called ideal w.r.t. $(P, \preceq)$ if $i \in I, j \preceq i$ implies $j \in I$. An element $i \in P$ is called lower neighbour of $j \in P$ if $i \prec j$ and there exists no $k \in P$ with $i \prec k \prec j$. Given a lattice $(L, \preceq, \wedge, \vee)$ an element $i \in L$ is called join-irreducible if for every $j$ and $k$ in $L$ it holds that $i=j \vee k$ implies $i=j$ or $i=k$.

Distributive lattices. A lattice $(L, \preceq, \wedge, \vee)$ is called distributive if the binary operators $\wedge, \vee$ satisfy the distributive law $i \wedge(j \vee k)=(i \wedge j) \vee(i \wedge k)$ for all $i, j, k \in L$. (Note that this implies that also $i \vee(j \wedge k)=(i \vee j) \wedge(i \vee k)$ holds.) Alternatively, distributive lattices can be characterized by the exclusion of the sublattices $N_{5}$ and $M_{3}$ (see Figure 1).

Birkhoff's representation of distributive lattices. By a theorem of Birkhoff [1], a finite distributive lattice $L=(L, \preceq, \wedge, \vee)$ admits a canonical representation as a unionand intersection-closed system of sets in the following way: Let $P \subseteq L$ denote the set of all join-irreducible elements of $L$. Then the ordering $\preceq$ in $L$ induces the poset $P=(P, \preceq)$. Let $\mathscr{D}(P)$ denote the collection of all ideals w.r.t. $(P, \preceq)$. Then there exists an order isomorphism $\phi: L \rightarrow \mathscr{D}(P)$ between the two lattices $(L, \preceq, \wedge, \vee)$ and $(\mathscr{D}(P), \subseteq, \cap, \cup)$ with $\phi(i)=\{p \in P \mid p \preceq i\}$ and $\phi^{-1}(I)=\bigvee\{p \in P \mid p \in I\}$. For further details on lattices, the reader is referred to [1].

Distributive lattice polyhedra. Let us call a lattice polyhedron distributive if $(A, L)$ is of modular characteristic and lattice $L$ is distributive. One important example of distributive lattice polyhedra are Edmonds-Giles polyhedra: 


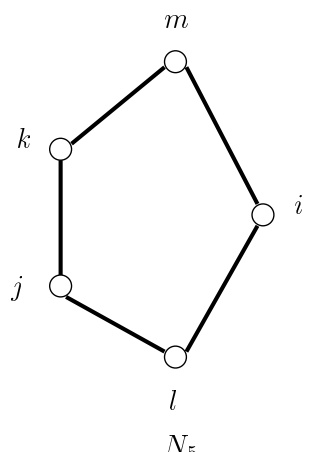

$N_{5}$

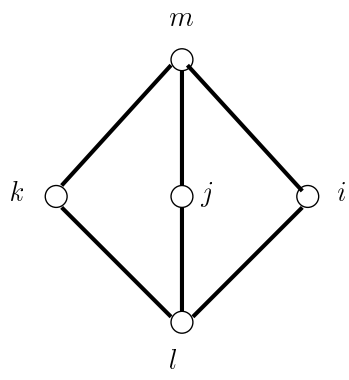

$M_{3}$

Fig. 1 Lattices $N_{5}$ and $M_{3}$.

Edmonds-Giles polyhedra and the submodular flow problem. Independent of the lattice polyhedron model, Edmonds and Giles introduced the submodular flow problem [3], i.e., the problem to optimize a linear function over an Edmonds-Giles polyhedron as a common generalization of the min-cost flow problem, the polymatroid intersection, and the minimum directed-cut covering problem:

Let $G=(V, E)$ be a connected directed graph and $\mathscr{F} \subseteq 2^{V}$ be a ring family, i.e., a set family that is closed with respect to the set-theoretic intersection and union. Note that $(\mathscr{F}, \subseteq, \cap, \cup)$ is a distributive lattice. Given a submodular function $f: \mathscr{F} \rightarrow \mathbb{R}$, an edge-assignment $x \in \mathbb{R}^{|E|}$ is called a submodular flow if

$$
x\left(\Delta^{+}(S)\right)-x\left(\Delta^{-}(S)\right) \leq f(S) \forall S \in \mathscr{F},
$$

where $\Delta^{+}(S)$ and $\Delta^{-}(S)$ denote, respectively, the sets of arcs leaving $S$ and entering $S$. Edmonds and Giles [3] proved that the linear inequality system in (1) is box-TDI, i.e., the system is TDI even if we add box-constraints $c \leq x \leq d$ for any integral lower and upper bound capacities $c, d \in \mathbb{Z}^{E}$. The polyhedron

$$
\mathbb{P}_{\mathrm{EG}}(G, \mathscr{F}, f)=\left\{x \in \mathbb{R}^{E} \mid x\left(\Delta^{+}(S)\right)-x\left(\Delta^{-}(S)\right) \leq f(S) \forall S \in \mathscr{F}, c \leq x \leq d\right\},
$$

is called Edmonds-Giles polyhedron, and the corresponding linear optimization problem is called submodular flow problem $(S F)^{1}$. In the last decades, numerous algorithms have been developed for the submodular flow problem, which is said to be "one of the most important frameworks for efficiently solvable combinatorial optimization problems"“[20].

It is not hard to see that an Edmonds-Giles polyhedron belongs to the class of distributive lattice polyhedra: given an Edmonds-Giles polyhedron $\mathbb{P}_{\mathrm{EG}}(G, \mathscr{F}, f)$ consider the collection of ordered pairs $L=\left\{\left(\Delta^{+}(S), \Delta^{-}(S)\right) \mid S \in \mathscr{F}\right\} \subseteq 3^{E}$ partially

\footnotetext{
1 Originally, the submodular flow problem was defined in terms of crossing submodular functions on crossing families. It is, however, shown in [8] that is suffices to consider submodular functions on ring families.
} 
ordered by $\left(\Delta^{+}(S), \Delta^{-}(S)\right) \preceq\left(\Delta^{+}(T), \Delta^{-}(T)\right)$ if and only if $S \subseteq T$. Note that $(L, \preceq)$ forms a distributive lattice with meet and join operations corresponding to the cuts induced by the intersection and union of the two vertex sets in question. Also for all $S \subseteq V$ and $e \in E$ define

$$
\chi(S, e)=\left\{\begin{aligned}
1 & \text { if } e \in \Delta^{+}(S) \\
-1 & \text { if } e \in \Delta^{-}(S) \\
0 & \text { otherwise. }
\end{aligned}\right.
$$

Then $(L, \preceq, \wedge, \vee)$ together with the incidence matrix $A=(\chi(S, e))_{S \in \mathscr{F}, e \in E}$ is consecutive and of modular characteristic. Moreover, $\mathscr{F}$ (and thus $L$ ) is distributive. Thus, the integrality of Edmonds-Giles polyhedra follows as a consequence of Theorem 1.

Schrijver's reduction of distributive lattice polyhedra to Edmonds-Giles polyhedra.

Let $\mathbb{P}(L, f)$ be a distributive lattice polyhedron, i.e., $L$ is a consecutive distributive lattice of modular characteristic on which $f$ is submodular. Schrijver [21] showed that this model coincides with the Edmonds-Giles model via the following reduction: Consider the set $V$ of all join-irreducible elements of lattice $L$, which will turn out to be the vertices in the corresponding Edmonds-Giles model. For each $i \in L$ define the vertex set $V_{i}:=\{j \in V \mid j \preceq i\}$. Then $\mathscr{F}=\left\{V_{i} \mid i \in L\right\} \subseteq 2^{V}$ is a ring family on subsets of vertices. Now, pick any $e \in E$ (recall that we assumed that there exists at least one $i^{*} \in L$ with $\chi\left(i^{*}, e\right) \neq 0$, say $\left.\chi\left(i^{*}, e\right)=1\right)$. Since $L$ has modular characteristic, we know that there exist unique minimal and maximal elements $i_{e}$ and $M_{e}$ in the sublattice $L_{e}^{+}=\{i \in L \mid \chi(i, e)=1\}$. Schrijver showed that the set $\left\{j \in L \mid j \succ M_{e}\right\}$ contains a unique minimal element $j_{e}$. Furthermore, he proved that the two elements $i_{e}$ (as defined above as unique minimal element in $L_{e}^{+}$) and $j_{e}$ are both join-irreducible. Assigning the arc $a_{e}=\left(i_{e}, j_{e}\right)$ (in the case $\chi\left(i^{*}, e\right)=-1$ the arc gets opposite direction) one can show for each $i \in L$ that $a_{e}$ enters the vertex set $V_{i}$ iff $\chi(i, e)=-1$, and $a_{e}$ leaves $V_{i}$ iff $\chi(i, e)=1$. Thus, the distributive lattice polyhedron model coincides with the Edmonds-Giles model.

Schrijver's reduction of distributive lattice polyhedra to Edmonds-Giles polyhedra is a big step into the direction of understanding the combinatorial structure of lattice polyhedra in general, and finding a combinatorial algorithm for them. However, if we really want to transform his reduction into an algorithm to solve the problem, we would need an efficient way to construct the auxiliary digraph, i.e., we would need to identify for each $e \in E$ the minimal element $i_{e}$ and the maximal element $M_{e}$ in $L_{e}^{+}$(resp. $L_{e}^{-}=\{i \in L \mid \chi(i, e)=-1\}$ in the case $\chi\left(i^{*}, e\right)=-1$ ), as well as the minimal element $j_{e}$ in the set $\left\{j \in L \mid j \succ M_{e}\right\}$ (note that such an element $j_{e}$ always exists by our assumption that the unique maximal element $M$ satisfies $\chi(M, e)=0$ for all $e \in E$ ). Furthermore, Schrijver requires that lattice $L$ is not only of modular characteristic, but also distributive whereas Gröflin and Hoffman's integrality result holds for all $L$ of modular characteristic. (In fact, in [21], the requirement of $L$ being distributive seems to be forgotten. However, the distributivity is necessary for the correctness of the proof in [21].) So one of the first questions to answer is whether we can find a combinatorial algorithm for lattice polyhedra where we only require that $L$ has modular characteristic. 
Our contribution. In this paper we will see that the modular characteristic of $L$ is almost sufficient for the lattice polyhedron to be distributive (Theorem 2). It turns out that it suffices to require the additional property that $\chi(i) \neq \chi(j)$ whenever $i$ is a lower neighbor of $j$ in $(L, \preceq)$. We call this property $\left(^{*}\right)$. In Section 3.1 , we give an alternative, constructive proof to show that the inequality system of a distributive lattice polyhedron corresponds to one in an Edmonds-Giles polyhedron, and vice versa. That is, given a lattice polyhedron in which $L$ has modular characteristic and satisfies property (*), we give a simple way to construct an auxiliary digraph $G$ so that the lattice polyhedron is equivalent to some Edmonds-Giles polyhedron on $G$. We will see that in order to construct $G$, we only need to consider the elements of an arbitrary maximal chain in $L$.

\section{Distributive lattice polyhedra and Edmonds-Giles polyhedra}

Birkhoff's representation of the lattice elements by ideals in $(\mathscr{D}(P), \subseteq)$ as described above turns out to be the key for the reduction of distributive lattice polyhedra to Edmonds-Giles polyhedra in the subsequent Section 3.1. Before going into its detail, let us observe that the modular characteristic of $L$ (i.e., property (C3) is satisfied with equality) is almost sufficient for the lattice polyhedron to be distributive.

Theorem 2 Let $\mathbb{P}(A, f)$ be a lattice polyhedron where the underlying lattice L has modular characteristic and satisfies

$$
\chi(i) \neq \chi(j) \text { whenever } i \text { is a lower neighbor of } j \text { in }(L, \preceq) .
$$

Then the underlying lattice $(L, \preceq, \wedge, \vee)$ is distributive.

Proof For the sake of contradiction, assume that $L$ is not distributive, i.e., that it contains an $N_{5}$ - or an $M_{3}$-sublattice (see Figure 1). Then there exist five distinct elements $i, j, k, l, m \in L$ such that $l=i \wedge j=i \wedge k$ and $m=i \vee j=i \vee k$. Note that we can assume that $\mathrm{j}$ is a lower neighbor of $k$ in the lattice $L$ if $j$ and $k$ are elements in the $N_{5}$ sublattice, and that $j$ and $k$ are lower neighbors of $m$ in the lattice $L$ if $j, k$, and $m$ are elements in the $M_{3}$-sublattice. By $\left(^{*}\right)$, it follows immediately that $\chi(j) \neq \chi(k)$ must be true in the $N_{5}$-sublattice. However, $\chi(j) \neq \chi(k)$ must also be true in the $M_{3}$-sublattice as otherwise $\chi(j)=\chi(m)$ by the consecutivity and modular characteristic of $L$, in contradiction to (*). Thus, we can choose some element $e \in E$ with $\chi(j, e) \neq \chi(k, e)$. Since $L$ is of modular characteristic, it follows that $\chi(l, e)+\chi(m, e)=\chi(i, e)+\chi(j, e)=\chi(i, e)+\chi(k, e)$, which implies $\chi(j, e)=\chi(k, e)$, a contradiction.

\subsection{Reduction to Edmonds-Giles polyhedra}

Let $\mathbb{P}(A, f)$ (respectively $\mathbb{P}(L, f)$ ) be a distributive lattice polyhedron, and $P$ denote the set of join-irreducible elements of the underlying distributive lattice $(L, \preceq, \wedge, \vee)$. By Birkhoff's Theorem, we may identify each element $i \in L$ (resp. the corresponding ordered pair $\left(X_{i}, Y_{i}\right) \in 3^{E}$ with $X_{i}:=\{e \in E \mid \chi(i, e)=1\}$ and $Y_{i}:=\{e \in E \mid \chi(i, e)=$ 
-1\}) with some ideal $I_{i} \in \mathscr{D}(P)$. Thus, our inequality system $\left\{x \in \mathbb{R}^{E} \mid A x \leq f\right\}$ can be rewritten as $\left\{x \in \mathbb{R}^{E} \mid x\left(X_{I}\right)-x\left(Y_{I}\right) \leq f_{I}, \forall I \in \mathscr{D}(P)\right\}$. In the following, we will construct an auxiliary digraph $G=\left(P, E^{\prime}\right)$ whose vertices correspond to the joinirreducibles of $L$, and whose edges are copies of $E$, i.e., $E^{\prime}=\left\{e^{\prime} \mid e \in E\right\}$, such that for all $I \in \mathscr{D}(P)$

$$
\begin{aligned}
& e \in X_{I} \quad \Longleftrightarrow \quad e^{\prime} \in \Delta^{+}(I), \\
& e \in Y_{I} \quad \Longleftrightarrow \quad e^{\prime} \in \Delta^{-}(I) .
\end{aligned}
$$

This directly implies that the vectors in $\mathbb{P}(A, f)$ are exactly the vectors in the EdmondsGiles polyhedron $\mathbb{P}(G, \mathscr{D}(P), f)$.

Construction of auxiliary digraph. To construct our digraph corresponding to $\mathbb{P}(A, f)$, we arbitrarily choose a maximal chain $(\mathscr{C}, \preceq)=\left\{\left(X_{1}, Y_{1}\right) \prec\left(X_{2}, Y_{2}\right) \prec \cdots \prec\left(X_{n}, Y_{n}\right)\right\}$ in $(L, \preceq)$.

Observation 1 Each $e \in E$ occurs either in some set $\left\{X_{i}\right\}_{i \in P}$ or in some set $\left\{Y_{i}\right\}_{i \in P}$.

Proof By (C2), an element $e \in E$ cannot occur both in some $X_{i}$ and some $Y_{j}$ for $i, j \in$ $\{1, \ldots, n\}$. Suppose for the sake of contradiction that $e$ does not occur in any chain element $\left(X_{i}, Y_{i}\right) \in \mathscr{C}$ at all. Let $(X, Y)$ be the minimal element in $(L, \preceq)$ with $e \in X \cup Y$, and let $\left(X_{i}, Y_{i}\right)$ be the minimal element of chain $(\mathscr{C}, \preceq)$ with $(X, Y) \prec\left(X_{i}, Y_{i}\right)$. Then $(X, Y)$ and $\left(X_{i-1}, Y_{i-1}\right)$ must be incomparable. Since $e$ occurs neither in $\left(X_{i-1}, Y_{i-1}\right)$ nor in $\left(X_{i}, Y_{i}\right)=(X, Y) \vee\left(X_{i-1}, Y_{i-1}\right)$, the modular characteristic of $L$ implies that $e$ occurs in $(X, Y) \wedge\left(X_{i-1}, Y_{i-1}\right)$. But, since $(X, Y) \wedge\left(X_{i-1}, Y_{i-1}\right) \prec(X, Y)$, this is a contradiction to the choice of $(X, Y)$.

Note that, by Birkhoff's Theorem, our chosen chain $(\mathscr{C}, \preceq)$ is isomorphic to a chain $(\overline{\mathscr{C}}, \subseteq)=P_{0} \subset P_{1} \subset P_{2} \subset \cdots \subset P_{n}$ in $(\mathscr{D}(P), \subseteq)$, where $P_{n}=\left\{p_{1}, \ldots, p_{n}\right\}$ is a linear extension of $\left(P, \preceq\right.$ ) (i.e., $p_{i} \prec p_{j}$ implies $i<j$ ), and $P_{i-1}=P_{i} \backslash\left\{p_{i}\right\}$ for $i=$ $1, \ldots, n$. We now define for all $e \in E$ the potential $v_{e}: P \rightarrow\{-1,0,1\}$ with $v_{e}\left(p_{i}\right)=$ $\chi\left(P_{i}, e\right)-\chi\left(P_{i-1}, e\right)$ for all $i=1, \ldots, n$. By the consecutiveness of $L$ we observe that each $v_{e}$ uniquely defines $i_{e}, j_{e}$ with $v_{e}\left(i_{e}\right)=1$ and $v_{e}\left(j_{e}\right)=-1$. These vertices $i_{e}$ and $j_{e}$ will now form the tail and head of $\operatorname{arc} e^{\prime}$, i.e., we define the $\operatorname{arc}$ set $E^{\prime}=\left\{e^{\prime}=\right.$ $\left.\left(i_{e}, j_{e}\right) \mid e \in E\right\}$. (See Figure 2.)

Theorem 3 If $\mathbb{P}(A, f)$ is a distributive lattice polyhedron and $G=\left(P, E^{\prime}\right)$ is the auxiliary digraph described above, then $\mathbb{P}(A, f)=\mathbb{P}_{E G}(G, \mathscr{D}(P), f)$.

Proof Note that the statement of the theorem follows immediately if we can show that for all ideals $I \in \mathscr{D}(P)$ with the corresponding lattice element $\left(X_{I}, Y_{I}\right) \in L$ and all $e \in E$ the equivalences (2) and (3) hold. Note that these are equivalent to

$$
\begin{gathered}
\chi(I, e)=1 \Longleftrightarrow e^{\prime} \in \Delta^{+}(I), \\
\chi(I, e)=-1 \Longleftrightarrow e^{\prime} \in \Delta^{-}(I) .
\end{gathered}
$$

Thus, in order to show the statement of Theorem 3 it suffices to prove that

$$
\chi(I, e)=\sum_{p \in I} v_{e}(p) \quad \forall I \in \mathscr{D}(P) \quad e \in E .
$$



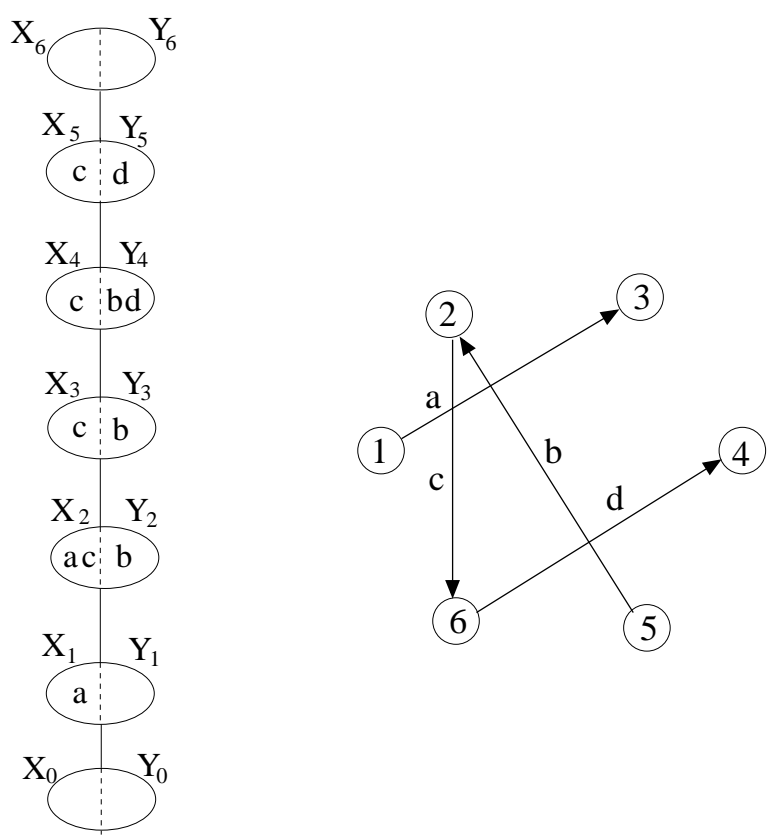

Fig. 2 A maximal chain in $L$ and the corresponding auxiliary graph.

This follows as a consequence of Lemma 7.5 in [9]. For the sake of completeness, we add the proof of (6) here: For any integers $1 \leq i_{1}<\cdots<i_{m} \leq n$ consider $I=\left\{p_{i_{1}}, \ldots, p_{i_{m}}\right\}$ with $\left\{p_{i_{k}}\right\}=P_{i_{k}} \backslash P_{i_{k}-1}(k=1, \ldots, m)$. Since $\chi(\cdot, e)$ is a modular function, it follows that

$$
\begin{aligned}
& \chi(I, e)+\sum_{k=1}^{m} \chi\left(P_{i_{k}-1}, e\right) \\
& =\chi(\underbrace{I \cup P_{i_{m}-1}}_{P_{i_{m}}}, e)+\sum_{k=2}^{m} \chi(\underbrace{\left(I \cap P_{i_{k}-1}\right) \cup P_{i_{k-1}-1}}_{P_{i_{k-1}}}, e)+\chi(\underbrace{I \cap P_{i_{1}-1}}_{\emptyset}, e) \\
& =\sum_{k=1}^{m} \chi\left(P_{i_{k}}, e\right),
\end{aligned}
$$

which implies that

$$
\chi(I, e)=\sum_{k=1}^{m} \chi\left(P_{i_{k}}, e\right)-\sum_{k=1}^{m} \chi\left(P_{i_{k}-1}, e\right)=\sum_{p \in I} v_{e}(p) .
$$

Acknowledgements Our special thanks go to Satoru Iwata for his very helpful comments on our original constructive proof of Theorem 3. which lead us to a simpler one described in Section 3.1. Satoru Fujishige's work was partly supported by a Grant-in-Aid from the Ministry of Education, Culture, Sports and Technology of Japan. Britta Peis' work was supported by the German Research Foundation (DFG). 


\section{References}

1. G. Birkhoff. Lattice Theory, volume 91. American Mathematical Society, 1991.

2. B. L. Dietrich and A. J. Hoffman. On greedy algorithms, partially ordered sets, and submodular functions. IBM Journal of Research and Developments, 47:25-30, 2003.

3. J. Edmonds and R. Giles. A min-max relation for submodular functions on graphs. Annals of Discrete Mathematics, 1:185-204, 1977.

4. U. Faigle and W. Kern. Submodular linear programs on forests. Mathematical Programming, 72:195206, 1996.

5. U. Faigle and W. Kern. On the core of ordered submodular cost games. Mathematical Programming, $87: 483-489,2000$

6. U. Faigle and B. Peis. Two-phase greedy algorithms for some classes of combinatorial linear programs. In Shang-Hua Teng, editor, Proceedings of the Nineteenth Annual ACM-SIAM Symposium on Discrete Algorithms, SODA 2008, San Francisco, California, USA, SIAM, pages 161-166, 2008.

7. A. Frank. Increasing the rooted-connectivity of a digraph by one. Mathematical Programming, 84:565-576, 1999.

8. S. Fujishige. Structures of polyhedra determined by submodular functions on crossing families. Mathematical Programming, 29:125-141, 1984.

9. S. Fujishige. Submodular functions and optimization. Annals of Discrete Mathematics (2. edition), $58,2005$.

10. S. Fujishige and S. Iwata. Algorithms for submodular flows. IEICE Transactions on Information and Systems. (Special Issue on Algorithm Engineering: Surveys), E83-E:322-329, 2000.

11. H. Gröflin and A.J. Hoffman. Lattice polyhedra ii: Generalization, constructions and examples. Annals of Discrete Mathematics, 15:189-203, 1982.

12. A. J. Hoffman and D. E. Schwartz. On lattice polyhedra. In A. Hajnal and V. T. Sos, editors, Proceedings of Fifth Hungarian Combinatorial Coll., pages 593-598, 1978.

13. U. Krueger. Structural aspects of ordered polymatroids. Discrete Applied Mathematics, 99:125-148, 2000.

14. S. Iwata L. Fleischer and S. T. McCormick. A faster capacity scaling algorithm for minimum cost submodular flow. Mathematical Programming, 92:119-139, 2002.

15. F. Spieksma M. Queyranne and F. Tardella. A general class of greedily solvable linear programs. Mathematics of Operations Research, 23:892-908, 1998.

16. S. T. McCormick. Submodular function minimization. In G. Nemhauser K. Aardal and R. Weismantel, editors, Handbook on Discrete Optimization, Elsevier, pages 321-391, 2006.

17. S. T. McCormick and S. Fujishige. Strongly polynomial and fully combinatorial algorithms for bisubmodular function minimization. Mathematical Programming, 122:87-120, 2010.

18. S.T. McCormick and B. Peis. A primal-dual algorithm for weighted abstract cut packing. In Oktay Günlück and Gerhard Woeginger, editors, Integer Programming and Combinatoral Optimization 15th International Conference, IPCO 2011, New York, USA, volume 6655 of Lecture Notes in Computer Science, Springer, pages 324-335, 2011.

19. S. T. McCormick S. Iwata and M. Shigeno. A faster algorithm for minimum cost submodular flows. In Proceedings of 9th ACM-SIAM Symposium on Discrete Algorithms, pages 167-174, 1998.

20. S. T. McCormick S. Iwata and M. Shigeno. A fast cost scaling algorithm for submodular flow. Information Processing Letters, 74:125-148, 2000.

21. A. Schrijver. Total dual integrality from directed graphs, crossing families and sub and supermodular functions. In W.R. Pulleyblank, editor, Progress in Combinatorial Optimization, Academic Press, pages 315-362, 1984.

22. A. Schrijver. Combinatorial Optimization. Springer, 2003. 